\title{
BRIEF REPORT Malignant Triton Tumor in a Patient With Li-Fraumeni Syndrome and a Novel TP53 Mutation
}

\author{
Mwe Mwe Chao, MD, ${ }^{1}$ John E. Levine, $\mathrm{MD},{ }^{1}$ Robert E. Ruiz, MD, PhD, ${ }^{2}$ Wendy K. Kohlmann, $\mathrm{MS}^{3}$ \\ Matthew A. Bower, Ms, ${ }^{4}$ Elizabeth M. Petty, $\mathrm{MD}^{3}$ and Rajen J. Mody, MD ${ }^{1}$
}

We report a 3-year-old boy with a malignant triton tumor (MTT) involving the left masticator space with local invasion and regional lymph node metastasis. Family history and detection of a novel germline TP53 mutation confirmed his diagnosis of Li Fraumeni syndrome (LFS). MTT has not been previously described in association with LFS. This case along with a comprehensive review of the literature, illustrate the importance of both somatic and germline TP53 mutations in the pathogenesis MTT. The tumor could not be resected and he was successfully treated with intensive induction chemotherapy, irradiation, and high-dose chemotherapy with autologous stem cell transplantation. Pediatr Blood Cancer (c) 2005 Wiley-Liss, Inc

Key words: Li-Fraumeni syndrome; malignant peripheral nerve sheath tumor; malignant triton tumor; TP53

\section{INTRODUCTION}

Malignant triton tumor (MTT) is a subtype of malignant peripheral nerve sheath tumor (MPNST) characterized by the presence of rhabdomyosarcomatous elements in a background of Schwannoma cells [1,2]. The tumor presents as a painful or painless firm enlarging mass in a variety of locations including the trunk, extremities, and head and neck $[3,4]$. Often large and medium nerves such as the sciatic nerve, spinal root, lumbosacral plexus, brachial plexus, and cranial nerves are affected $[1,2]$. This rare tumor with fewer than 100 cases reported in the literature, generally affects adult patients but can also occur in children [5]. Although MTT can present as a sporadic tumor, approximately 60 $70 \%$ occurs in young patients with neurofibromatosis type 1 (NF1) [2]. The tumor is highly malignant with a 5-year survival rate of only $10-20 \%$ [2]. Outcome data is limited, but suggests that complete tumor resection appears to improve survival. A beneficial role for adjuvant chemotherapy and radiation has not been established [4].

We herein describe the occurrence of MTT in a patient with Li-Fraumeni syndrome (LFS). Although a variety of soft-tissue sarcomas occur in LFS patients, MTT has not been previously reported in this syndrome. A novel germline mutation in TP53 tumor suppressor gene was identified in this patient. Moreover, somatic mutations in p53 are frequently encountered in a majority of MPNST/MTT samples [6-10]. These findings implicate a role for p53 deregulation in the pathogenesis of this aggressive tumor. Surgical resection could not be undertaken in this patient and he was successfully treated with intensive chemotherapy for induction, irradiation for local control, and high-dose chemotherapy with autologous stem cell transplantation for consolidation. He is alive without evidence of disease recurrence at 43 months from diagnosis.

\section{CASE}

A 3-year-old boy was referred to the University of Michigan Pediatric Hematology-Oncology clinic after a 4-week history of an enlarging, painless swelling within the left cheek. There was no history of recent trauma or dental infection. The family history was significant for a sister diagnosed with low-grade adrenocortical carcinoma at 2 years of life, a mother with breast cancer at 36 years of age and a prior history of thyroid cancer in her 20s, a maternal grandmother with thyroid cancer in her 40s, and a maternal grandfather with skin cancers and lymphoma. There was also a family history of gastric cancer in a maternal great-aunt (Fig. 1).

On physical examination, the patient was normal in appearance. There was a firm, nontender mass measuring approximately $6 \mathrm{~cm}$ in diameter located within his left cheek. Under his left buccal mucosa was a white colored tumor. There was no palpable cervical lymphadenopathy. The patient did not have any physical stigmata of NF1 such as neurofibromas, café-au-lait spots, axillary or inguinal freckling, or Lisch nodules.

${ }^{1}$ Department of Pediatric Hematology-Oncology and Bone Marrow Transplantation, University of Michigan Health System, Ann Arbor, Michigan; ${ }^{2}$ Department of Pathology, University of Michigan Health System, Ann Arbor, Michigan; ${ }^{3}$ Internal Medicine-Molecular Medicine and Human Genetics, University of Michigan Health System, Ann Arbor, Michigan; ${ }^{4}$ Diagnostic Laboratory, University of Minnesota Medical Center-Fairview, Minneapolis, Minnesota

*Correspondence to: Dr. Mwe Mwe Chao, Department of Pediatrics, Division of Pediatric Hematology-Oncology, University of Michigan Health System, 1500 East Medical Center Drive, CCGC B1-207, Ann Arbor, MI 48109-0914. E-mail: mmchao@umich.edu

Received 9 September 2005; Accepted 18 October 2005 


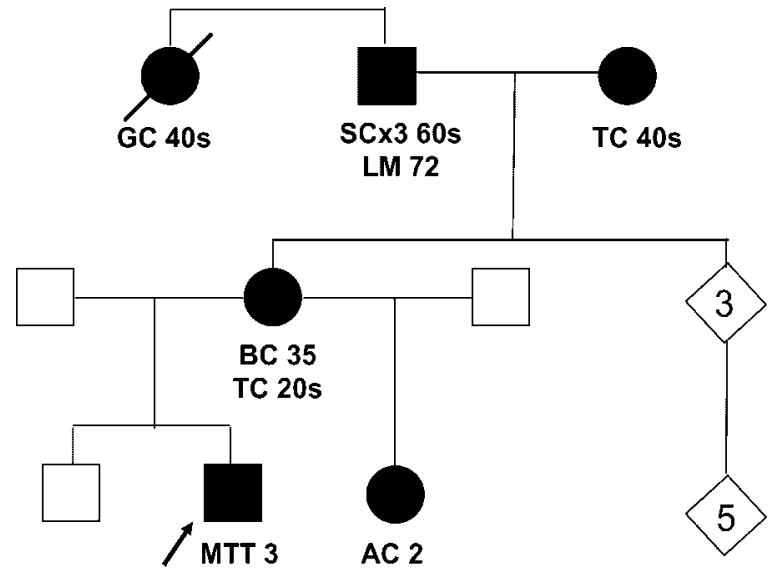

Fig. 1. A classic Li-Fraumeni syndrome pedigree showing multiple family members with cancers, a young age at onset of malignancies, and multiple primary cancers in an affected individual. The proband (arrow) was tested and found to have a TP53 mutation. Other family members have not been tested. It is unclear from the family history whether the mutation originated from the maternal grandfather or maternal grandmother. Squares, males; Circles, females; Diamond with numbers, individuals of unspecific sex; Shaded, diagnosed with cancer; Slash, deceased; GC, gastric cancer; SC, skin cancer; LM, lymphoma; TC, thyroid cancer; BC, breast cancer; MTT, malignant triton tumor; AC, adrenocortical carcinoma. Numbers indicate age at tumor diagnosis.

Maxillofacial and neck CT scans demonstrated a $5.5 \times 3 \times 5 \mathrm{~cm}$ mass centered in the left masticator space with extension and invasion into the left maxillary sinus, lateral oral cavity, parapharyngeal fat space, and orbital space. Destruction of multiple facial bones was also noted (Fig. 2A). There was no intracranial extension. Enlarged pathologic appearing lymph nodes in the region of the left submandibular gland were present. Staging body CT scans, bone scan, and bone marrow biopsies were negative for distant metastatic disease.

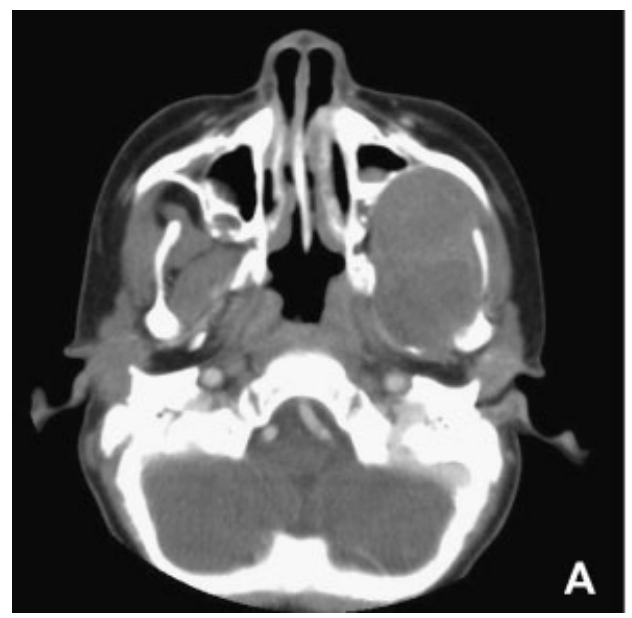

Fig. 2. Axial images from maxillofacial computed tomography scans. (A) At diagnosis the large tumor invaded and eroded through adjacent structures. (B) Three months after autologous stem cell transplant, the primary tumor had significantly decreased in size (arrows). A residual mass, which is stable in size and PET negative remains and is presumed to be scar tissue.

Pediatr Blood Cancer DOI 10.1002/pbc

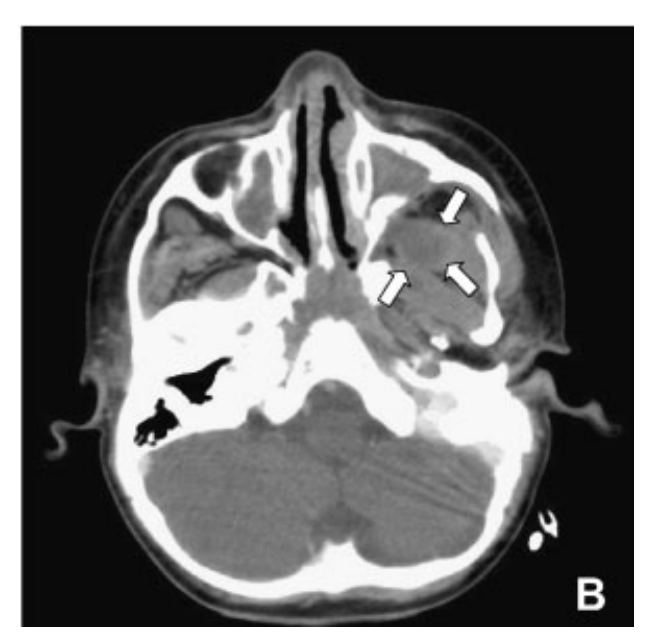

Biopsy and histologic examination of the tumor revealed a malignant neoplasm composed of intersecting fascicles of variably pleomorphic spindle cells. The malignant cells showed predominantly oval nuclei with granular chromatin and prominent nucleoli (Fig. 3A). In selected areas, markedly enlarged and occasional multinucleated cells were observed (Fig. 3B). Immunohistochemical stains showed subsets of tumor cells positive for S-100 protein, including the most pleomorphic and multinucleated cells (Fig. 3C). Another subset of tumor cells, generally less pleomorphic, was positive for actin HHF-35 (Fig. 3D). Scattered tumor cell nuclei were positive for myogenin. The malignant cells were negative for melanoma marker Melan-A, epithelial membrane antigen, and pan keratin. These immunohistologic findings established the diagnosis of MTT.

To test for a TP53 mutation, a blood specimen was sent to the University of Minnesota Medical Center-Fairview Diagnostic Laboratory. Leukocyte DNA was extracted and polymerase-chain-reaction (PCR) was used to amplify exons 4-10 of the gene. PCR amplified DNA fragments were sequenced using an Applied Biosystems 3100 analyzer. Comparison of the patient's sequence with the wild type TP53 sequence showed a deletion of GT at 686-687 in exon 7. This heterozygous frameshift mutation is predicted to introduce a premature stop codon at amino acid 238 by altering the preceding 229-237 amino acids.

The tumor was deemed unresectable and the patient received intensive induction chemotherapy, consisting of courses of vincristine, doxorubicin, and cyclophosphamide alternating with ifosfamide and etoposide administered every 3 weeks over 48 weeks. The doxorubicin was co-administered with dexrazoxane for cardio-protection. The patient also received 70.2 Gy of radiation for local control at week 12. As consolidation therapy, the patient was enrolled on a clinical research study using high-dose chemotherapy with

Pediatr Blood Cancer DOI 10.1002/pbc 

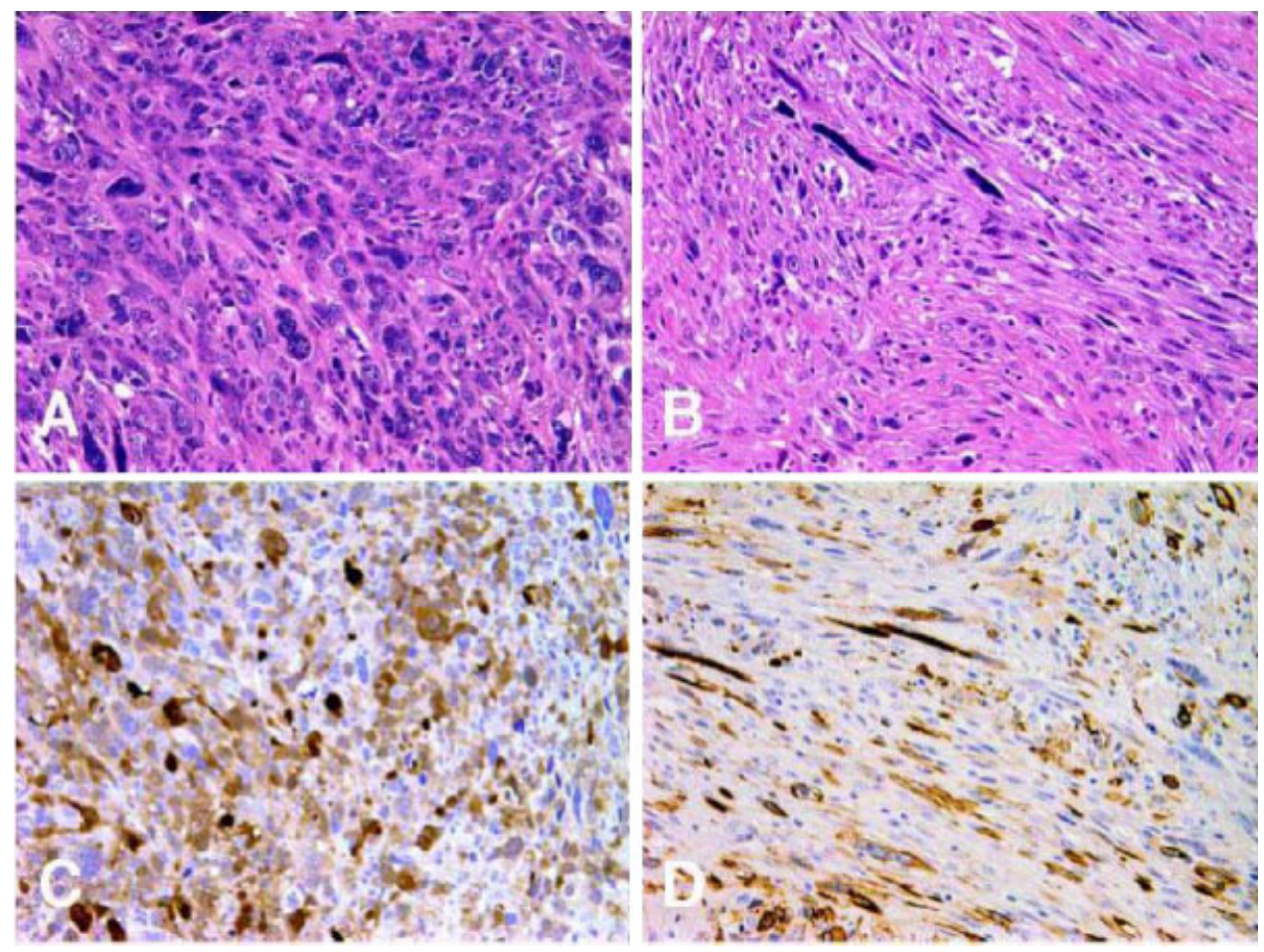

Fig. 3. $(H \& E, 200 \times)($ A) Pleomorphic and multinucleated spindle cells comprise a portion of this neoplasm. (B) Variably pleomorphic but generally more regular spindle cells comprise the majority of the neoplasm. (Immunoperoxidase, $200 \times$ ) (C) S-100 protein immunostain is positive in a small subset of spindle cells and especially in the most atypical and multinucleated cells, identifying the MPNST component. (D) Muscle actin HHF-35 is positive in a larger subset of spindle cells and occasional pleomorphic cells, identifying the rhabdomyosarcoma component.

thiotepa and carboplatin followed by autologous stem cell rescue for poor-prognosis, rare cancers. The patient tolerated the therapies well without significant complications. His end of therapy evaluation showed an $85 \%$ reduction in size of the primary tumor (Fig. 2B). Forty-three months from diagnosis, the patient continues in remission.

\section{DISCUSSION}

LFS is a rare autosomal dominant cancer predisposition syndrome. It is a highly penetrant disorder associated with a $50 \%$ cancer risk by age 40 years and up to $90 \%$ by age 60 [11]. LFS patients often develop multiple primary cancers at a young age. Additionally, these patients are at risk for a wide range of malignancies, with a particularly high occurrence of osteosarcomas, soft-tissue sarcomas, premenopausal breast cancer, brain tumors, adrenocortical tumors, and acute leukemias [11-14]. A variety of other cancers have been reported at a lower rate in LFS patients. In this report, we describe a pediatric patient with MTT and LFS with a novel TP53 tumor suppressor gene mutation. Although the association of MTT with a germline TP53 mutation in humans has not been observed previously, our experience and a review of the literature suggest that deregulation of p53 may be important in the pathophysiology of MPNST/MTT [6-10].
TP53 tumor suppressor gene mutations are commonly encountered in human cancers. Over $60 \%$ of families with LFS have identifiable germline mutations in this gene [14]. Additionally, in some LFS patients and families with a clinical diagnosis of LFS where a TP53 mutation is not detected, germline mutations in the $C H K 2$ gene that encodes a protein product involved in p53 phosphorylation can be found [15]. Sporadic tumors likewise frequently $(>50 \%)$ harbor TP53 mutations and the presence of such mutations often denote a more aggressive phenotype [7,8,14]. The TP53 gene located at chromosome band $17 \mathrm{p} 13$ spans $20 \mathrm{~kb}$ and consists of one noncoding and ten coding exons. The protein product is 393 amino acids in length with five highly conserved domains. Functionally, the N-terminal region of the protein contains a strong transcriptional activation domain while the $\mathrm{C}$-terminal region has a tetramerization domain. In the intervening region, there is a sequencespecific DNA binding domain (Fig. 4) [14,16]. The p53 protein forms a tetramer and serves as a transcription activator of downstream targets involved in cell growth, differentiation, and proliferation. Cellular stress and DNA damage induces p53 accumulation within the nucleus to cause cell cycle arrest and/or apoptosis [14]. A large number of mutations have been identified throughout the gene, however, the majority are missense mutations occurring within the DNA-binding domain in exons 5-8 [14,15]. These 
A

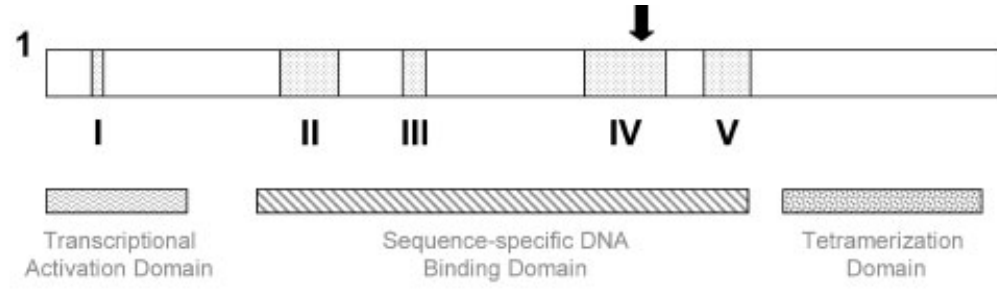

B

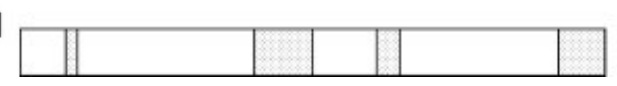

Fig. 4. (A) Basic structure of the p53 protein consisting of 393 amino acids. Five highly conserved domains (I-IV) confer distinct functions as described in the text. Solid arrow shows the location of the frameshift mutation in our patient resulting in a truncated protein $(\mathbf{B})$. The tetramerization domain is lost.

mutations result in reduced protein function and an inability to maintain genomic integrity.

Our patient's leukocytes harbored a heterozygous two base pair deletion, GT, at nucleotides 686-687 in exon 7 of the TP53 gene. A search through the p53 and LFS mutational databases revealed that this deletional mutation has not been previously observed. It is predicted to create a frameshift, mutation altering amino acids 229-237 and introducing a premature stop codon at amino acid 238. Interestingly, a similar but not identical mutation (a deletion of two base pairs at codons 209-210 that resulted in a premature stop codon at amino acid 214 in exon 6) was reported by Toguchida et al. in an 8-year-old girl with malignant fibrous histiocytoma, a soft tissue sarcoma superficially resembling MPNST, and a family history of tumors of neural-tissue origin [17]. Based on models by Vogelstein et al., we speculate that the truncation of $40 \%$ of the C-terminus of the protein in our patient as a consequence of the deletional mutation will not allow for oligomerization, resulting in reduction of p53 tetramers, and decreased expression of growth inhibitory genes and malignant transformation (Fig. 4) [18].

A number of other studies have previously implicated TP53 deregulation in MPNST/MTT malignant transformation. Tumor samples from NF1-related and NF1-unrelated MPNST/MTT cases have TP53 gene missense mutations, loss of heterozygosity, or p53 nuclear accumulation $[7,8,10]$. Leroy et al. and Zhou et al. showed that highgrade MPNST had strong nuclear immunoreactivity for p53 while neurofibromas did not, suggesting that nuclear accumulation of p53 may herald progression to malignancy $[7,8]$. More recently a causal role for p53 mutation and MPNST/MTT development has emerged. Berghmans et al. reported that zebrafish with missense germline mutations in the DNA-binding domain of tp53 developed MPNST at a high rate. Embryos that harbored this mutation, failed to undergo apoptosis in response to $\gamma$ radiation. The mutant fish also failed to upregulate p21, a downstream target of p53, and did not arrest at the G1/S checkpoint after irradiation. These reports and our current case study, together suggest that mutations in p53 appear to play a role in the genesis of MPNST/MTT.

Clinically MTT is a highly malignant neoplasm with a dismal prognosis and a 10-20\% survival rate at 5 years [2]. Optimal management remains to be established because the rarity of diagnosis has precluded a disease-specific longitudinal study. However, review of the literature shows that like other soft tissue sarcomas, patients with MTT who undergo a complete tumor resection fared better than those who undergo incomplete resection [4,19]. Our patient presented with a large MTT involving his left masticator space with local invasion and regional metastatic disease. Surgical expiration of the tumor would have been highly morbid and, therefore, was not feasible for our patient. We elected to treat him with induction chemotherapy designed for Ewing's sarcoma because the therapy included doxorubicin and ifosfamide, agents known to be active against soft tissue sarcomas generally and specifically against rhabdomyosarcoma and tumors of neural origin (doxorubicin for the rhabdomyosarcoma component and ifosfamide for the malignant Schwann cells). Radiation therapy was administered during the chemotherapy course for local control and to maximize dose intensity for our patient, we used high-dose chemotherapy with autologous stem cell rescue for consolidation. Our patient remains without evidence of disease at 43 months from diagnosis. Although this approach proved beneficial for our patient, the anecdotal nature of this finding limits its clinical application. Additionally, this approach was undertaken for our patient with the recognition that this therapy in the context of a germline p53 mutation as seen in LFS may significantly increase the risk for a second malignancy. Our patient, like other LFS patients, will require annual comprehensive medical examinations.

In conclusion, we describe a 3-year-old boy with MTT who was found to have a novel germline mutation in TP53. Although the molecular mechanisms leading to the development of MPNST/MTT have not been fully elucidated, our findings support a role for p53 deregulation in MPNST/MTT 
tumorigenesis. In patients presenting with MPNST/MTT, physicians should not only look for physical signs of NF1, but also elicit a detailed medical and family history to evaluate for LFS. Clinically, the treatment of MPNST/MTT remains a challenge especially when the tumor cannot be completely excised. Our patient's tumor responded to intensive chemotherapy which included autologous hematopoietic stem cell transplantation and local irradiation. Given the aggressive nature of this tumor, this therapeutic strategy may be beneficial for other patients with MPNST/MTT as adjuvant therapy to improve survival or neoadjuvant therapy to facilitate tumor removal. This approach, however, should be done in the setting of a clinical research study for rare aggressive sarcomas.

\section{ACKNOWLEDGMENT}

Special thanks to Dr. Christian Kratz and Dr. Dan Wechsler for their helpful suggestions and review of this manuscript and Dr. Peter Strouse for providing the radiographic images.

\section{REFERENCES}

1. Kleihues P, Cavenee WK, editors. Pathology and Genetics of Tumors of the Nervous System. Lyon: International agency for research on cancer; 1997. pp 130-132.

2. Miettinen M. Diagnostic soft tissue pathology. Washington, DC: Churchill Livingstone; 2003. pp 367-378.

3. Baehring JM, Betensky RA, Batchelor TT. Malignant peripheral nerve sheath tumor: The clinical spectrum and outcome of treatment. Neurology 2003;61:696-698.

4. Victoria L, McCulloch TM, Callaghan EJ, et al. Malignant triton tumor of the head and neck: A case report and review of the literature. Head Neck 1999;21:663-670.
5. Kim ST, Kim CW, Han GC, et al. Malignant triton tumor of the nasal cavity. Head Neck 2001;23:1075-1078.

6. Haddadin MH, Hawkins AL, Long P, et al. Cytogenetic study of malignant triton tumor: A case report. Cancer Genet Cytogenet 2003;144:100-105.

7. Zhou H, Coffin CM, Perkins SL, et al. Malignant peripheral nerve sheath tumor: A comparison of grade, immunophenotype, and cell cycle/growth activation marker expression in sporadic and neurofibromatosis 1-related lesions. Am J Surg Pathol 2003;27: $1337-1345$.

8. Leroy K, Dumas V, Martin-Garcia N, et al. Malignant peripheral nerve sheath tumors associated with neurofibromatosis type 1: A clinicopathologic and molecular study of 17 patients. Arch Dermatol 2001;137:908-913.

9. Magrini E, Pragliola A, Fantasia D, et al. Acquisition of i(8q) as an early event in malignant triton tumors. Cancer Genet Cytogenet 2004; $154: 150-155$.

10. Strauss BL, Gutmann DH, Dehner LP, et al. Molecular analysis of malignant triton tumors. Hum Pathol 1999;30:984-988.

11. Schneider K. Li-Fraumni Syndrome. Accessed December 14, 2004. http://www.genetests.org

12. Jones G. Li-Fraumeni Syndrome. Accessed May 22, 2005. http:// www.emedicine.com/ped/topic 1305.htm

13. Varley JM. Germline TP53 mutations and Li-Fraumeni syndrome. Hum Mutat 2003;21:313-320.

14. Malkin D. The role of p53 in human cancer. J Neurooncol 2001; 51:231-243.

15. Chompret A. The Li-Fraumeni syndrome. Biochimie 2002;84: $75-82$.

16. National Cancer Institute. Tumor Protein p53; TP35. Online Mendelian Inheritance in Man. Accessed May 31, 2005. http:// www.ncbi.nlm.nih.gov/entrez/query.fcgi

17. Toguchida J, Yamaguchi T, Dayton SH, et al. Prevalence and spectrum of germline mutations of the p53 gene among patients with sarcoma. N Engl J Med 1992;326:1301-1308.

18. Vogelstein B, Kinzler KW. p53 function and dysfunction. Cell 1992;70:523-526.

19. deCou JM, Rao BN, Parham DM, et al. Malignant peripheral nerve sheath tumors: The St. Jude Children's Research Hospital experience. Ann Surg Oncol 1995;2:524-529. 